\title{
Comparative Study between the Effect of two Anesthetic Agents and Techniques on the Immune System
}

E.F.Gadalla, I.S.Abdelazem, A.A.Mosad and E.M.Mohamed

Anesthesia and Intensive Care Benha, Faculty of Medicine, Benha Univ., Benha, Egypt

E-Mail:dr.eman.m.salah@gmail.com

\begin{abstract}
General anesthesia and surgical stress have a major role in affecting patients' cellular immunity. Different anesthetic techniques may have different effects on the immune response of patients undergoing the same type of surgery. Comparing the effects of two types of anaesthesia on immunological and neurohormonal responses to anaesthesia and surgical stress. Forty patients were assigned to undergo either volatile induction or maintenance of anaesthesia [VIMA] with sevoflurane or total intravenous anaesthesia [TIVA] with propofol. Plasma cortisol, glucose and CRP levels were measured, A blood sample was collected $24 \mathrm{~h}$ before surgery [Sbasal] to achieve the basal findings of the patient. The first sample on the day operation was collected just before the induction of anesthesia [S1], the second sample was collected $2 \mathrm{~h}$ later after induction [S2] and the third sample was collected $24 \mathrm{~h}$ after surgery [S3]. VIMA with sevoflurane is less immunosuppressive than TIVA with propofol in patients undergoing surgery. Further studies will be needed to ensure that these results could help anesthetists to select safer anesthetic circumstances especially for immunocompromised patients performing different types of surgeries. TIVA reduce stress hormone responses compared with VIMA. This result suggest that TIVA with propofol may has advantages over VIMA with sevoflurane in stress related responses to surgery , including secretion of cortisol and glucose.
\end{abstract}

Keywords: Anathesia, Immune system, TIVA, VIMA.

\section{Introduction}

The immune system is a host defense system comprising many biological structures and processes within an organism that protects against disease. To function properly, an immune system must detect a wide variety of agents, known as pathogens, from viruses to parasitic worms, and distinguish them from the organism's own healthy tissue. In many species, there are two major subsystems of the immune system: the innate immune system and the adaptive immune system. Both subsystems use humoral immunity and cell-mediated immunity to perform their functions [1]. General anesthesia and surgical stress have a major role in affecting patients' cellular immunity . Different anesthetic techniques may have different effects on the immune response of patients undergoing the same type of surgery [2].

Since both anesthesia and surgery affect the immune system in many ways, a large number of studies have been paying concern to the perioperative immune responses . Moreover, the effect of anesthetics perse on the immune system has taken a lot of attention [3]. Drugs commonly used in anesthesia and intensive care may modulate immunological reactions by influencing intracellular communication through modification of cytokine response and fluctuation of peripheral immune cells such as natural killer [NK] cells, B cells, and T lymphocyte subpopulations [ CD4 and CD8 cells] [4].

Potent stimuli including surgery, trauma and anaesthesia induce systemic endocrine, immunological and metabolic responses resulting in increased lipolysis, proteolysis and impaired glucose utilization. Surgery provokes hemodynamic, metabolic, and inflammatory responses; it also provokes a complex immune reaction that includes activation of the interleukin network [5]. For example, surgery and anesthesia provoke an increase in proinflammatory interleukins and adhesion molecules, and a subsequent increase in countervailing anti- inflammatory interleukins. The most important proinflammatory interleukin is IL-6, while the most potent anti-inflammatory interleukin is IL-10 [6].

Volatile induction and maintenance of anaesthesia [VIMA] and total intravenous anaesthesia [TIVA] are techniques frequently used in ambulatory anaesthesia .Both provide safe anaesthesia and have the advantages of offering rapid emergence compared with conventional anaesthesia, together with a reduction in the number of drugs required for induction and maintenance. Propofol is a commonly used intravenous anesthetic which has been effectively used for induction and maintenance of anesthesia [TIVA] [7].

Sevoflurane is a general inhalational anesthetic that can be used for inhalational induction and maintenance of general anesthesia due to its non-irritant and pleasant odor [VIMA] [8] .

Whether inflammatory responses to surgery are comparably activated during total intravenous anesthesia [TIVA] and during volatile anesthesia remains unclear [9].

The aim of the present study is to compare the effects of two anesthetic techniques : total intravenous anesthesia [TIVA] with propofol versus volatile induction and maintenance anesthesia [VIMA] with sevoflurane on immunological and neurohormonal responses in patients undergoing different types of surgeries.

\section{Patients and methods}

\subsection{Sample size}

Sample size was calculated according to a pilot study of the first 5 patients for the primary outcome [level of CRP after 2 hours] Assuming that the power of the study was $80 \%$ and $\alpha$ error was 0.05 the calculated effect size was 1.0068. 17 patients in each group were found to be satisfactory . we considered 20 patients in each group to overcome the dropout. 
Patients were divided into 2 equal groups Group [1] :TIVA group [Group T: $\mathrm{n}=20]$. Group [2] :VIMA group [Group V:n=20].

\subsection{Inclusion criteria}

Patients of both sexes, Ages between 18-60 years.

\subsection{Exclusion criteria}

Preexisting chest diseases, Diabetes mellitus, Renal, hepatic and cerebrovascular diseases and Patients with preoperative signs of infection.

\section{4 study design}

A blood sample was collected $24 \mathrm{~h}$ before surgery [Sbasal] to achieve the basal findings of the patient. The first sample on the day operation was collected just before the induction of anesthesia [S1], the second sample was collected $2 \mathrm{~h}$ later after induction [S2] and the third sample was collected $24 \mathrm{~h}$ after surgery [S3]. Samples were analyzed for Plasma cortisol, glucose and CRP levels.

\subsection{Ethical considerations}

Ethical committee approval from Benha university hospitals was taken and written informed consent was obtained from every patient before enrollment in the study.

\subsection{Anesthetic techniques}

In all cases the surgery was performed as the first operation of the day in the same time in order to eliminate diurnal variations in circulating stress hormones. On arrival at the operating room , an 18-gauge catheter was inserted in the antecubital vein of the opposite arm to the side where surgery was to be done . For all patients four venous blood samples were collected each sample is $5 \mathrm{ml}$ in a single tube.

\subsection{Intraoperative monitoring}

Arterial $\mathrm{O} 2$ saturation, end-tidal $\mathrm{CO} 2$, inspired and end-tidal sevoflurane concentrations, haemodynamic variables including heart rate and blood pressure, were monitored in all patients.

For TIVA, anaesthesia was induced and maintained with propofol and neuromuscular blocker [Atracurium Besylate], for VIMA using sevoflurane and neuromuscular blocker [Atracurium Besylate ]. Patients were given consecutive numbers, those with odd numbers receive TIVA while those with even numbers receive VIMA.

All patients were premedicated with $0.1 \mathrm{mg} / \mathrm{kg}$ midazolam and $0.5 \mathrm{mg}$ atropine intramuscularly $1 \mathrm{~h}$ before surgery. All patients were also given a loading dose of fentanyl $1 \mathrm{Mg} / \mathrm{kg}$ i.v. and $100 \%$ oxygen via a face mask for 2-3 min before induction. Supplementary doses of fentanyl were administered intraoperatively as required.
In Group $\mathrm{T}$, induction was performed using propofol $1-2.5 \mathrm{mg} / \mathrm{kg}$ and anesthesia was maintained with propofol $4-8 \mathrm{mg} / \mathrm{kg} / \mathrm{h}$.

In Group $\mathrm{V}$, induction was done using a face mask with sevoflurane starting at $8 \%$ with an initial fresh gas flow [FGF] of $6 \mathrm{~L} / \mathrm{min}$ for 1-3 min and reaching down to a FGF of $3 \mathrm{~L} / \mathrm{min}$ during maintenance together with 1.5$3.5 \%$ sevoflurane .

Atracurium $0.6 \mathrm{mg} / \mathrm{kg}$ i.v. was administered to facilitate tracheal intubation in both groups Muscle relaxation was maintained using incremental doses of Atracurium $10 \mathrm{mg}$ boluses as required .

After skin closure, abrupt discontinuation of either sevoflurane or propofol and reversal of residual muscle relaxation was done with Neostigmine and Atropine and the lungs were ventilated with $100 \%$ oxygen at a flow rate of at least $6 \mathrm{~L} / \mathrm{min}$.

\subsection{Data management and statistical method}

Data management and statistical analysis were done using SPSS vs.25. [IBM, Armonk, New York, United states]. Numerical data was summarized as means and standard deviations. Categorical data was summarized as numbers and percentages. Comparisons between two groups were done using Mann Whitney $U$ test for numerical data. Categorical data was compared using Chi-square test. Markers were compared within groups using Friedman's test. Pairwise analysis was done in case of significant overall effect. All P values were two sided. All pairwise analysis were adjusted for multiple comparisons. $\mathrm{P}$ values less than 0.05 were considered significant.

\section{Results}

Forty patients were assigned to undergo either volatile induction and maintenance of anaesthesia [VIMA] with sevoflurane or total intravenous anaesthesia [TIVA] with propofol

Patients were divided into 2 equal groups :Group [1] :TIVA group [Group T: $\mathrm{n}=20$ ] and Group [2] :VIMA group [Group V:n=20]

There was no significant difference between two groups as regard age \& gender. P values were 0.986 \& 0.327 respectively. Mean duration of surgery was significantly higher in group I [120 min] compared to group II $[101 \mathrm{~min}]$. $\mathrm{P}$ value $<0.001$. Mean duration of anesthesia was significantly higher in group I [157 min] compared to group II [142 min]. P value $<0.001$. There were no significant differences between two groups as regard weight \& height. P values were $0.620 \& 0.565$ respectively Table (1).

Regarding CRP [figure1]: CRP levels was significantly higher in group I than group II at S1 and S3. At $\mathrm{S} 1$ mean CRP was $5.89 \mathrm{mg} / 1$ in group I compared to $5.02 \mathrm{mg} / \mathrm{l}$ in group II. P value was $<0.001$. At S3 mean CRP was $12.53 \mathrm{mg} / \mathrm{l}$ in group I compared to $10.3 \mathrm{mg} / \mathrm{l}$ in group II. $\mathrm{P}$ value was $<0.001$. There were no significant differences between two groups at $\mathrm{S}$ basal and S2. P values were $0.738 \& 0.429$ respectively. 
Table (1) General characteristics in both groups

\begin{tabular}{lccc}
\hline & Group I & Group II & P value \\
\hline Age [Years], & $33 \pm 7$ & $33 \pm 10$ & 0.986 \\
Gender, Males n[\%] & $9[45.0]$ & $6[30.0]$ & 0.327 \\
Weight[kg]mean \pm SD & $80 \pm 9$ & $79 \pm 9$ & 0.62 \\
Height cm, mean \pm SD & $166 \pm 7$ & $166 \pm 5$ & 0.565 \\
$\begin{array}{l}\text { Duration of } \\
\text { surgery,min }\end{array}$ & $120 \pm 5$ & $101 \pm 7$ & $<0.001$ \\
$\begin{array}{l}\text { Duration of } \\
\text { anesthesia,mins }\end{array}$ & $157 \pm 8$ & $142 \pm 7$ & $<0.001$ \\
\hline
\end{tabular}

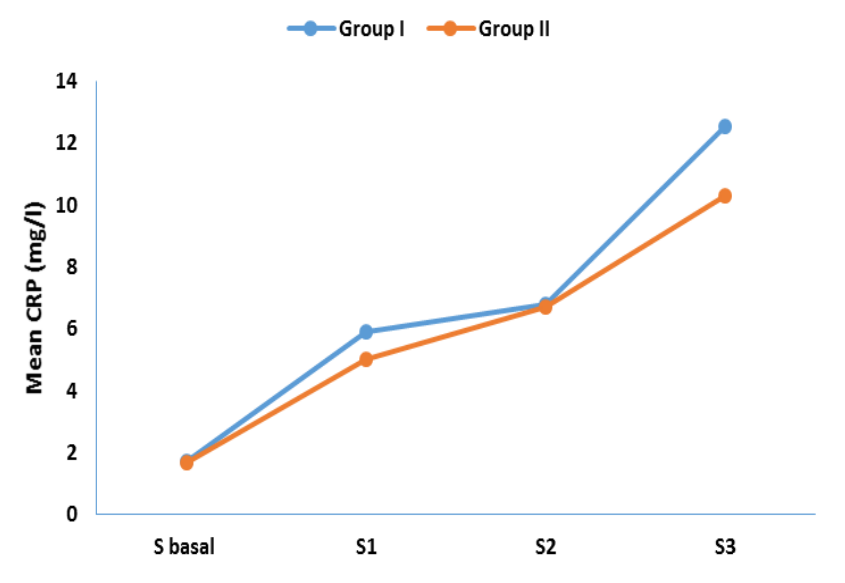

Fig (1) Mean CRP levels within each group over different times.

Regarding Cortisol levels between groups at different time points Fig (2)At $\mathrm{S}$ basal, mean cortisol was significantly higher in group II $[18.1 \mu \mathrm{g} / \mathrm{dl}]$ compared to group I [14.5 ug/dl]. P value was <0.001. At S1, mean cortisol was significantly higher in group II $[19.9 \mu \mathrm{g} / \mathrm{dl}$ compared to group I $[14.7 \mu \mathrm{g} / \mathrm{dl}]$. P value was $<0.001$.
At S2, mean cortisol was significantly higher in group II $[18 \mu \mathrm{g} / \mathrm{dl}]$ compared to group I [13.6 $\mu \mathrm{g} / \mathrm{dl}]$. P value was $<0.001$. At S3, mean cortisol was significantly higher in group II $[33 \mu \mathrm{g} / \mathrm{dl}]$ compared to group I [14.8 $\mu \mathrm{g} / \mathrm{dl}]$. P value was $<0.001$.

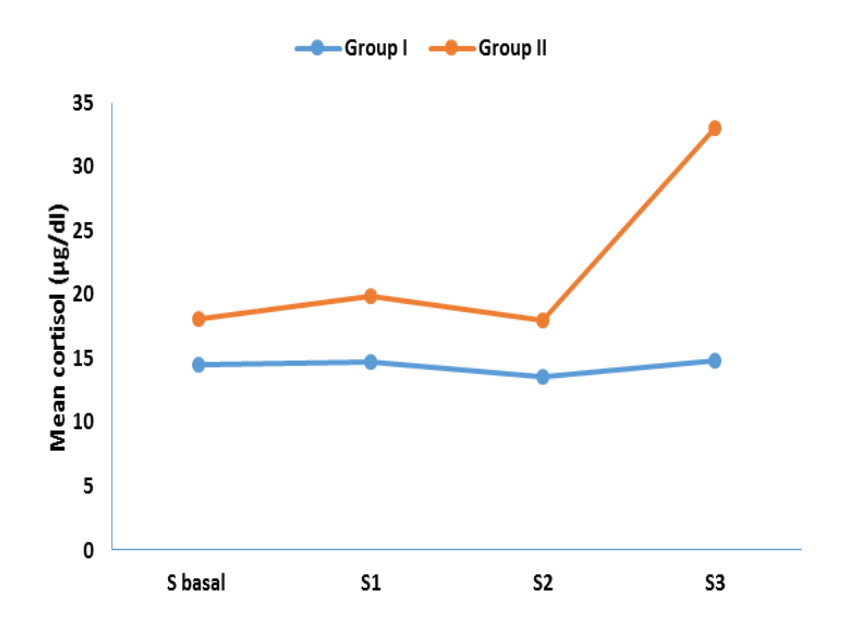

Fig (2) Mean cortisol levels within each group over different times.

Regarding Glucose levels between groups at different time points[Figure 3]: Glucose levels was significantly higher in group II than group I at S2 and S3. At S2 mean glucose was $98 \mathrm{mg} / \mathrm{dl}$ in group II compared to $85 \mathrm{mg} / \mathrm{dl}$ in group I. P value was $<0.001$. At S3 mean glucose was
$133 \mathrm{mg} / \mathrm{dl}$ in group II compared to $92 \mathrm{mg} / \mathrm{dl}$ in group I. P value was $<0.001$. There were no significant differences between two groups at $\mathrm{S}$ basal and $\mathrm{S} 1$. P values were $0.904 \& 0.841$ respectively. 


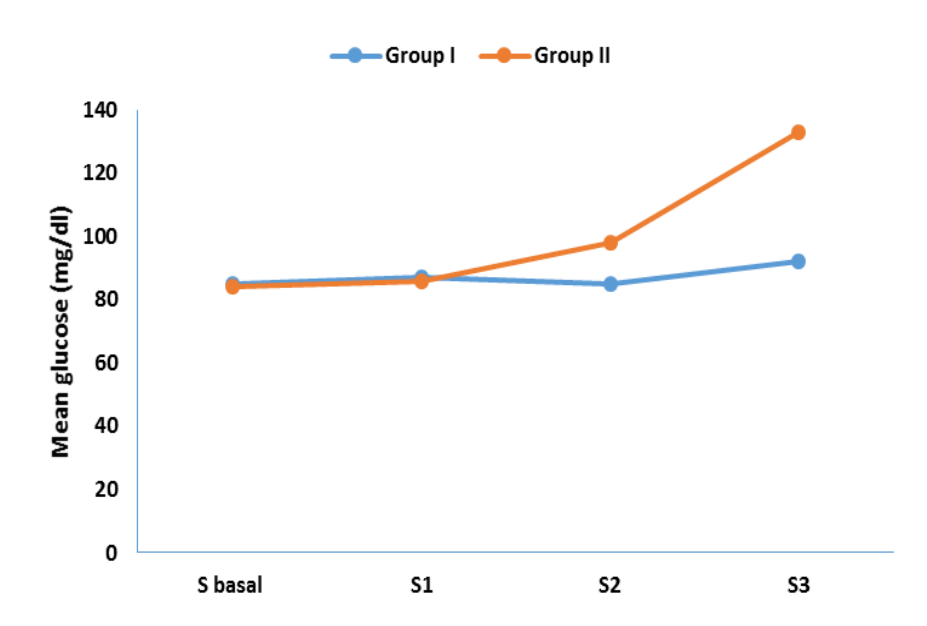

Fig (3) Mean glucose levels within each group over different times.

\section{Discussion}

There may be too many factors that might lead to the impairment of postoperative immunity when anesthesia and surgery are combined; including systemic illness, pathology involved, length of surgery, anesthetics and anesthetic techniques [10]. VIMA with sevoflurane produced less immune suppressive effects than TIVA with propofol. In the current study, Both techniques lead to immune-suppression, as shown by increased Creactive protein [CRP]. However, this immunesuppression was more prominent with propofol compared to sevoflurane .

Surgical tissue injury, anaesthesia and post-operative pain can alter the metabolic, neuroendocrine and immune systems [11] by affecting a variety of pro-inflammatory cytokines, including tumour necrosis factor $\alpha$ [TNF- $\alpha$, IL-1 and IL-6. These mediators are also responsible for stimulating the secretion of stress hormones, thereby rendering the patient's haemodynamic status unstable [12].

Alterations in acute phase reactants [APR] might occur in response to systemic inflammation which occurs in association with many conditions such as infection or trauma, Normal values of $\mathrm{C}$ reactive protein [CRP] may be $0-1.0 \mathrm{mg} / \mathrm{dL}$ [13]. The effect of anesthesia on acute phase reactants was evaluated in many studies with controversial results [14].

Elisena and coworkers [15] compared the effect of anesthesia with sevoflurane to propofol in one lung ventilation [OLV], their study showed significant increase in CRP levels with propofol compared to attenuate and non-significant increase in CRP with sevoflurane. They even suggested a possible antiinflammatory effect of sevoflurane.

In the current study, CRP was elevated in both groups , yet it was more significantly elevated with propofol compared to sevoflurane .

The effects of anaesthesia and surgery on the immune response are closely associated with patient outcomes. While appropriate inflammatory reactions are advantageous and essential for wound healing and host defence against microorganisms, excessive immune responses can be detrimental [5].
A study by Mustafa and colleagues [16] thought that the immune suppression produced by TIVA with propofol is related to the high lipid content of propofol preparations and may be attributed to its release of cytokines. However, this study differs from the current study in that we compared total intravenous general anesthesia with propofol versus total inhalation general anesthesia with sevoflurane, while that study compared two intravenous agents [ propofol vs thiopental ].

However, another study was not in agreement with the results obtained in the current study. In that study compared the immunological effects of sevoflurane were compared to that of propofol in patient undergoing laparoscopic cholecytectomy. They found that propofol group was accompanied by a higher ratio of CD4/CD8 and lower cortisol levels indicating that propofol might even exert an immune protective effect.This study might be criticized for small sample size [14 patients in each group] which might not be enough to support such conclusions. The mission of the human immune system is to defend the body against exterior invasion. In order to perform this function properly, the immune system must be able to distinguish between the subject's own cells and other invading organisms. This function can be done through the molecules of the major histocompatibility complex [MHC] [10].

Increases in cortisol and glucose levels are common features of patients with surgical stress but typically values rapidly return to normal [17]. Hyperglycaemia can however be accompanied by various adverse systemic effects including altered immune function and poorer neurological outcomes and may be detrimental in immunocompromised or neurologically unstable patients [18].

Although VIMA produced higher glucose levels compared with TIVA at all measured time points in the present study, neither technique was effective in attenuating intraoperative increases in glucose from baseline.

Cortisol levels are known to increase with increasing severity of surgical injury [19], TIVA produced a significantly lower level of cortisol than VIMA in the present study. In addition cortisol increased from 
baseline to emergence from anaesthesia in the VIMA group, whereas this increase was effectively attenuated by TIVA. Thus, in the present study, it was shown that the choice of anaesthetic technique may affect cortisol and glucose levels.

\section{Conclusion}

Volatile induction and maintenance of anaesthesia [VIMA] and total intravenous anaesthesia [TIVA] are techniques frequently used in ambulatory anaesthesia. Both provide safe anaesthesia and have the advantages of offering rapid emergence compared with conventional anaesthesia, together with a reduction in the number of drugs required for induction and maintenance. VIMA with sevoflurane is less immunosuppressive than TIVA with propofol in patients undergoing surgery however, TIVA with propofol may has advantages over VIMA with sevoflurane in stress related responses to surgery .

\section{References}

[1] B.Beck- Schimmer, Protection of pharmacological postconditioning in liver surgery: results of a prospective randomized controlled trial," Ann. Surg, Vol. 256( 5), PP. 837-845, 2012.

[2] Y. Hori, T. Ibuki, T. Hosokawa, and Y. Tanaka, "The effects of neurosurgical stress on peripheral lymphocyte subpopulations," J. Clin. Anesth, Vol. 15(1), PP. 1-8, 2003.

[3] M. Bauer: "Anesthesia and perioperative immune function. Remarks on the work of M. Bauer, $\mathrm{H}$. Rensing and T. Ziegenfuss [Anaesthesist 1998, 47: 538-556]," Anaesthesist, Vol. 47(11), p. 939, 1998.

[4] J.-M. Brand, C. Frohn, J. Luhm, H. Kirchner, and P. Schmucker, "Early alterations in the number of circulating lymphocyte subpopulations and enhanced proinflammatory immune response during opioidbased general anesthesia," Shock, Vol. 20(3), PP. 213-217, 2003.

[5] E. Lin, S. E. Calvano, and S. F. Lowry, "Inflammatory cytokines and cell response in surgery," Surgery, Vol. 127(2), PP. 117-126, 2000.

[6] W. T. McBride, M. A. Armstrong, and S. J. McBride, "Immunomodulation: an important concept in modern anaesthesia," Anaesthesia, Vol. 51(5), PP. 465-473, 1996.

[7] I. Smith, "Propofol. An update on its clinical use," Anesthesiology, Vol. 81, PP. 1005-1043, 1994.

[8] N. R. Puig , "Effects of sevoflurane general anesthesia: immunological studies in mice," Int. Immunopharmacol., Vol. 2(1), PP. 95-104, 2002.
[9] A. Koenig, U. D. Koenig, R. Heicappel, and H. Stoeckel, "Differences in lymphocyte mitogenic stimulation pattern depending on anaesthesia and operative trauma: I. Halothane-nitrous oxide anaesthesia.," Eur. J. Anaesthesiol., Vol. 4(1), PP. 17-24, 1987.

[10]N. TUNA, M. TUNA, D. KOCA, Ş. ATICI, and A. ARIBOĞAN, "The effects of three neurosurgical anaesthetic methods on HLA-DR expression," Turkish J. Med. Sci., Vol. 32( 1), PP. 65-71, 2002.

[11] M. Salo, "Effects of anaesthesia and surgery on the immune response," Acta Anaesthesiol. Scand., Vol. 36(3), PP. 201-220, 1992.

[12] W. S. Walker and H. A. Leaver, "Immunologic and stress responses following video-assisted thoracic surgery and open pulmonary lobectomy in early stage lung cancer," Thorac. Surg. Clin., Vol. 17(2), PP. 241-249, 2007.

[13] C. Gabay and I. Kushner, "Acute-phase proteins and other systemic responses to inflammation," N. Engl. J. Med., Vol. 340(6), PP. 448-454, 1999.

[14] V. Brix-Christensen, E. Tønnesen, I. J. Sørensen, T. V Bilfinger, R. G. Sanchez, and G. B. Stefano, "Effects of anaesthesia based on high versus low doses of opioids on the cytokine and acute-phase protein responses in patients undergoing cardiac surgery," Acta Anaesthesiol. Scand., Vol. 42(1), PP. 63-70, 1998.

[15]E. De Conno et al., "Anesthetic-induced improvement of the inflammatory response to onelung ventilation," Anesthesiol. J. Am. Soc. Anesthesiol, Vol. 110, (6), PP. 1316-1326, 2009.

[16] S. H. Waly, H. A. Baqi, Y. M. Nasr, N. M. A. Alazizi, and M. Atfy, "Immunomodulation: TIVA versus VIMA,” Egypt. J. Anaesth., Vol. 27(4), PP. 241-247, 2011.

[17] P. Christensen, M. R. Brandt, J. Rem, and H. Kehlet, "Influence of extradural morphine on the adrenocortical and hyperglycaemic response to surgery," Br. J. Anaesth, Vol. 54, (1), PP. 23-27, 1982.

[18] W. T. Longstreth Jr and T. S. Inui, "High blood glucose level on hospital admission and poor neurological recovery after cardiac arrest," Ann. Neurol. Off. J. Am. Neurol. Assoc. Child Neurol. Soc., Vol. 15(1), PP. 59-63, 1984.

[19] C. Traynor and G. M. Hall, "Endocrine and metabolic changes during surgery: anaesthetic implications," BJA Br. J. Anaesth., Vol. 53(2), PP. 153-160, 1981. 